\title{
The Osteoprotegerin - Von Willebrand Factor Complex: Protagonist Or Bystander In Atherothrombosis?
}

\author{
Nagy Elod ${ }^{*}$ \\ University of Medicine and Pharmacy of Tirgu Mures, Romania, Faculty of Pharmacy \\ Two important cardiovascular risk factors, osteoprotegerin and Von Willebrand Factor form complex in endothelial cells and in circulating \\ blood. The pathophysiological role of the complex is unknown. We give a brief review of the relevant literature and confront it with some of our \\ recent findings regarding the OPG-VWF correlation.
}

Keywords: osteoprotegerin, Von Willebrand Factor

Received: 22 January 2016 / Accepted: 24 January 2016

\section{To the editor:}

Endothelial dysfunction characterizes all of the main manifestations of atherosclerosis: coronary artery disease, carotid stenosis and peripheral arterial disease. The endothelium in atherosclerosis shifts to a pro-coagulant, cell-harboring, secretory biological surface with specific molecular patterns. Atherothrombosis is one of the most important complications of the vulnerable atheromatous plaque, and Von Willebrand Factor (VWF), a multimeric glycoprotein that mediates the adherence of platelets to the denudated endothelial surface, is implicated in this process (1). The grade of multimerization, and the activitity of its degrading protease, " a disintegrin and metalloproteinase with a thrombospondin type 1 motif, member 13 " (ADAMTS-13) largely influence VWF's biological activity (2).

Osteoprotegerin (OPG) is a cytokine produced in many cell types, with a variety of biological roles: regulates the homeostasis of the bone, mediates cell survival, prevents T-cell mediated inflammatory reactions (3). OPG is expressed throughout the cardiovascular system and can be detected in the heart tissue, the arterial wall, but also in veins (3). In human venous endothelial cells, osteoprotegerin, together with VWF and P-selectin resides in the secretory granules called the Weibel-Palade bodies. The OPG binding site on the VWF A1 domain has been identified and the co-secretion of the two molecules from the endothelial cells was documented $(4,5)$. Each of the two molecules increase in sera of patients with unstable coronary artery disease and critical limb ischemia, conditions with a high atherothrombotic risk (6-8). The circulating fraction of osteoprotegerin has a yet unknown commitment. OPG possibly exerts a protective role in the complex, but this still remains a hypothesis. Vinholt et al developed an enzyme-linked immunosorbent assay (ELISA) to determine

* Correspondence to: Elod Nagy

E-mail: elod.nagy@umftgm.ro the circulating OPG-VWF complexes and found that their levels do not correlate with the grade of coronary calcification in coronary artery disease patients (9). However, this is a single observation that cannot rule out the pathological importance of the OPG-VWF complex.

In a cohort of peripheral arterial disease patients $(n=105)$, we observed that cases with a positive history for acute myocardial infarction $(\mathrm{n}=11)$ have a stronger correlation of plasma VWF and OPG, than those without infarction. Moreover, patients with a previous stroke history $(\mathrm{n}=12)$ also possessed stronger VWF-OPG correlations, than those with no stroke (Nagy E et al, unpublished data). Since the subgroups were with a limited number, we have to interpret these findings carefully; however, due to the plenty of data concerning the cardiovascular risk factor role of osteoprotegerin, the association is worthwhile to be investigated on larger patient cohorts.

\section{Conflict of interest}

The authors hereby declare no conflict of interest.

\section{References}

1. Bryckaert M, Rosa JP, Denis CV, Lenting PJ- Of von Willebrand factor and platelets. Cell Mol Life Sci. 2015;72:307-326.

2. McKinnon TA, Chion AC, Millington AJ, Lane DA, Laffan MA- N-linked glycosylation of VWF modulates its interaction with ADAMTS13. Blood. 2008;111:3042-3049.

3. Reid P, Holen I - Pathophysiological role of osteoprotegerin (OPG). European Journal of Cell Biology. 2009;88:1-17.

4. Shahbazi S, Lenting PJ, Fribourg C, Terraube V, Denis CV, Christophe OD - Characterization of the interaction between von Willebrand factor and osteoprotegerin. J Thromb Haemost. 2007;5:1956-1962.

5. Zannettino AC, Holding CA, Diamond $P$ et al - Osteoprotegerin (OPG) is localized to the Weibel-Palade bodies of human vascular endothelial cells and is physically associated with von Willebrand factor. J Cell Physiol. 2005, 204:714-723.

6. Rutten B, Maseri A, Cianflone D - Plasma levels of active Von Willebrand factor are increased in patients with first ST-segment elevation myocardial infarction: A multicenter and multiethnic study. Eur Heart J Acute Cardiovasc Care. 2015;4:64-74.

7. Hosbond SE, Poulsen TS, Diederichsen ACP, Nybo M, Rasmussen LM, Mickley $\mathrm{H}$ - Osteoprotegerin as a marker of atherosclerosis: A systematic 
update. Scandinavian Cardiovascular Journal. 2012:46:203-211.

8. Ali Z, Ellington AA, Mosley TH, Jr., Kullo IJ: Association of serum osteoprotegerin with ankle-brachial index and urine albumin: creatinine ratio in African-Americans and non-Hispanic whites. Atherosclerosis. 2009;206:575-580
9. Vinholt PJ, Overgaard M, Diederichsen AC, Mickley $H$, Poulsen TS, Sand NP, Nybo M, Rasmussen LM: An ELISA for the quantitation of von Willebrand factor: osteoprotegerin complexes in plasma. Thromb Res. 2013,131:396-400. 